\title{
Periodismo ambiental en Canal Sur Televisión
}

\author{
Miguel Montaño Montaño \\ Grupo de Investigación en Estructura, Historia y Contenidos de la Comunicación \\ Periodista de Canal Sur TV
}

El autor estudia el origen histórico del periodismo ambiental, la evolución en España y Andalucía y su aparición en Canal Sur TV. Realiza un análisis sobre la información medioambiental, especialidad del periodismo que en la década de los noventa despierta nuevamente gran interés entre el público. La televisión autonómica ha dedicado, desde su creación, numerosos programas sobre el medio ambiente. Actualmente sus Servicios Informativos tienen la sección Medio Ambiente. De la importancia que se concede a este tipo de información es un buen ejemplo el programa «Tierra y Mar», que incorpora progresivamente noticias medioambientales, referidas a la educación ambiental, el mantenimiento y recuperación de la biodiversidad y la presentación de modelos de conducta respetuosos.

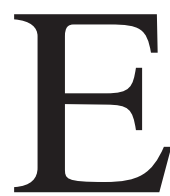

1 interés del público por los asuntos relacionados con el medio ambiente comenzó a desarrollarse entre la década de los sesenta y setenta. Posteriormente, este interés decayó y ahora, en los noventa, ha resurgido con fuerza la cuestión medioambiental. La cobertura de los medios de comunicación de los problemas medioambientales ha seguido, básicamente, la misma tendencia ${ }^{1}$.

Como en tantas otras cuestiones, la explosión informativa sobre cuestiones relacionadas con el medio ambiente tiene su origen en Estados Unidos, país donde se dejaron ver los primeros efectos del desarrollismo. En 1969, millones de norteamericanos de clase media habían huido del centro de las ciudades en busca de una nueva vida en suburbios ajardinados. Para su desaliento, se encontraron con que les seguían la contaminación y los congestionamientos. Estos ciudadanos constituyeron el primer núcleo de

\footnotetext{
MAUDIE, Kuns; NIESKE Witlox. «La comunicación y el medio ambiente». Revista de ciencias de la información. Número extraordinario, 1995. Facultad de Ciencias de la Información. Universidad Complutense de Madrid. Pág. 13.
} 
demanda de información sobre contaminantes y contaminadores ${ }^{2}$. Junto a este fenómeno, en los años sesenta surgen en los Estados Unidos los primeros movimientos ecologistas en el seno de las universidades en un ámbito pacifista, de libertad sexual y de acercamiento a la naturaleza. "En Europa, sin embargo, este movimiento tiene tintes subversivos pero como requerimiento de transformación del sistema hacia un modelo socialista ${ }^{3}$ " "En España los primeros grupos colectivos ecologista eclosionaron al calor de la frustración de mayo del 68 o de la mano del entonces activo movimiento ciudada$n o^{4}$ ".

El movimiento ecologista surge como un grito de protesta para la defensa del entorno natural y cultural ante la agresión de un tipo de desarrollo que se manifiesta en impactos como la contaminación industrial o urbana de ríos, mares o zonas naturales. La labor desempeñada por el movimiento ecologista, en los medios de comunicación a partir de la década de los setenta es un fenómeno poco estudiado. Una de las reflexiones sobre este tema la encontramos en El Libro de la Naturaleza, (El País, 1984) "libro de referencia imprescindible ${ }^{5}$ ". Un artículo de la bióloga y periodista Rosa P. Abelló ${ }^{6}$ Ulloa ofrece una visión general del protagonismo de las asociaciones ecologista en los medios

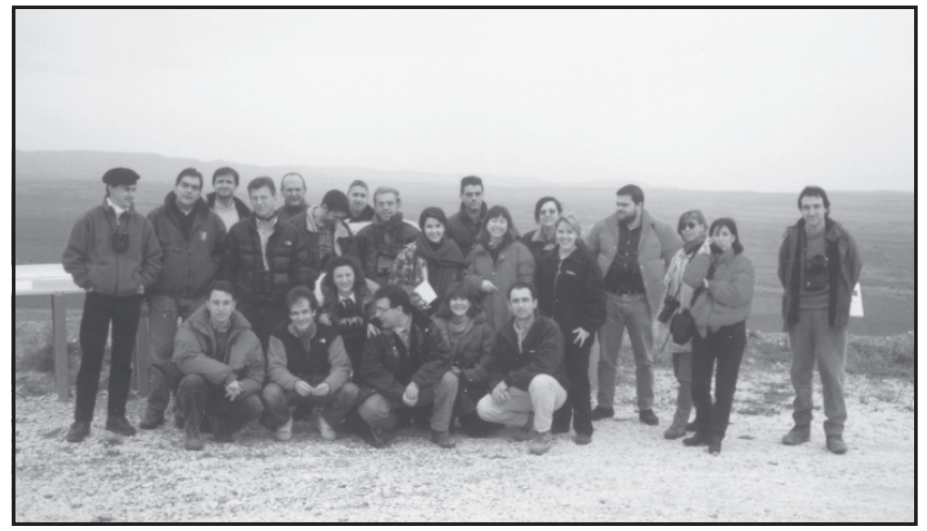

Periodistas especializados en medio ambiente de España durante unas jornadas organizadas por la Sociedad Española de Ornitología en Gallocanta, Zaragoza, en febrero de 1998. Una gran parte de los periodistas pertenecen a la Asociación de Periodistas de Información Ambiental de España. En estas jornadas participó un equipo de Canal Sur Televisión.

2 CALVO HERNANDO, Manuel. Actas del I Congreso Nacional de Periodismo Ambiental. Madrid. Noviembre, 1995. Pág. 120.

3 VERDÚ, Vicente. Actas inéditas del II Congreso Nacional de Periodismo Ambiental. Madrid. Noviembre, 1997.

4 SANZ CAÑADA, Luis A. «En España el movimiento ecologista empieza a ser historia». El libro de la naturaleza. Madrid. El País 1994, pág. 286.

5 FERNÁNDEZ SÁNCHEZ, Joaquín. Periodismo Ambiental en España. Centro de Publicaciones, Secretaría General Técnica. Ministerio de Obras Públicas, Transportes y Medio Ambiental, 1995.

6 P. ABELlÓ UllOA. Rosa. «Ecología y medios de comunicación». El libro de la naturaleza. Madrid. El País, 1984, pág. 112. 
de comunicación: Los ecologistas hacían sus comunicados y los distribuían, personalmente, en las redacciones de los periódicos. Era ésta la única manera de acceder a un medio que hasta entonces no se había interesado por los temas ambientales. En el mejor de los casos, existía un contacto en la redacción que se encargaba de que la noticia entrara. Esos contactos, con el tiempo, llegaron a ser sustituidos por verdaderos expertos. En España existen hoy más de doscientos profesionales especializados en información ambiental. Una gran mayoría se agrupa en la Asociación de Periodistas de Información Ambiental, de la que forman parte tres profesionales de Canal Sur Televisión.

En Andalucía el movimiento ecologista, junto a la labor de algunos profesionales de la información, "contribuyen a afianzar la información ambiental 7 ". El periodismo de información ambiental en Andalucía comienza en los primeros años de la década de los $80^{8}$. En estos momentos hay tres periodistas que, de una forma continuada, publican información ambiental. El diario $\mathrm{ABC}$ tiene cierto peso en este ámbito gracias a la labor del periodista Manuel Jesús Florencio que se dedicaba a temas exclusivos de medio ambiente y especializados en Doñana. En el caso del Diario Almería, el periodista especializado era Miguel Angel Blanco ${ }^{9}$. El tercero de los periodistas, trabaja hoy en Canal Sur Televisión, José María Montero.

\section{“Los verdes" de Canal Sur Televisión}

La programación de Canal Sur Televisión a lo largo de los 9 años de existencia de esta televisión autonómica ha dedicado numerosos programas, series y documentales a hablar sobre el medio ambiente andaluz desde las más diversas posiciones: naturalista, Parques Naturales; antropológica, Donde el viento da la vuelta, o recreativa, Andalucía Azul.

En un sentido estricto de información de actualidad especializada en medio ambiente destaca la reciente aparición de una sección en los Informativos Diario 1 y 2 denominada Medio Ambiente, hecho que ocurre con el cambio de programación efectuada en Febrero de 1997, así como el programa Tierra y Mar, que ha ido incorporando progresivamente informaciones de medio ambiente a lo largo de sus nueve años de existencia. "La evolución de la

\footnotetext{
MONTERO, José María. Periodista de Canal Sur Televisión. Premio Andalucía Medio Ambiente 1996. En declaraciones a quien suscribe este artículo. Sevilla, 7 de Noviembre 1997.

MONTERO, José María. Idem nota 7.

9 BLANCO, Miguel Angel. Premio Andalucía de Periodismo en 1989, por la sección de Ecología en el Diario Ideal de Granada. Entrevista realizada en Madrid, durante la celebración del II Congreso Nacional de Periodismo Ambiental en noviembre de 1997.
} 
información ambiental en Canal Sur ha sido similar a la de otros medios de comunicación. Dentro de la socorrida sección de sociedad y cultura se hace medio ambiente. La ventaja que había aquí desde un principio es que existía una persona, que era yo, que se ocupaba de estos temas y, por lo tanto, había una información continua ${ }^{10}$. En la actualidad este área depende orgánicamente de la sección de Sociedad y Cultura, a efectos de organizar los turnos de trabajo. En cuanto a contenidos, enfoques y previsión de temas, el periodista José María Montero es el único profesional que se dedica a estas tareas.

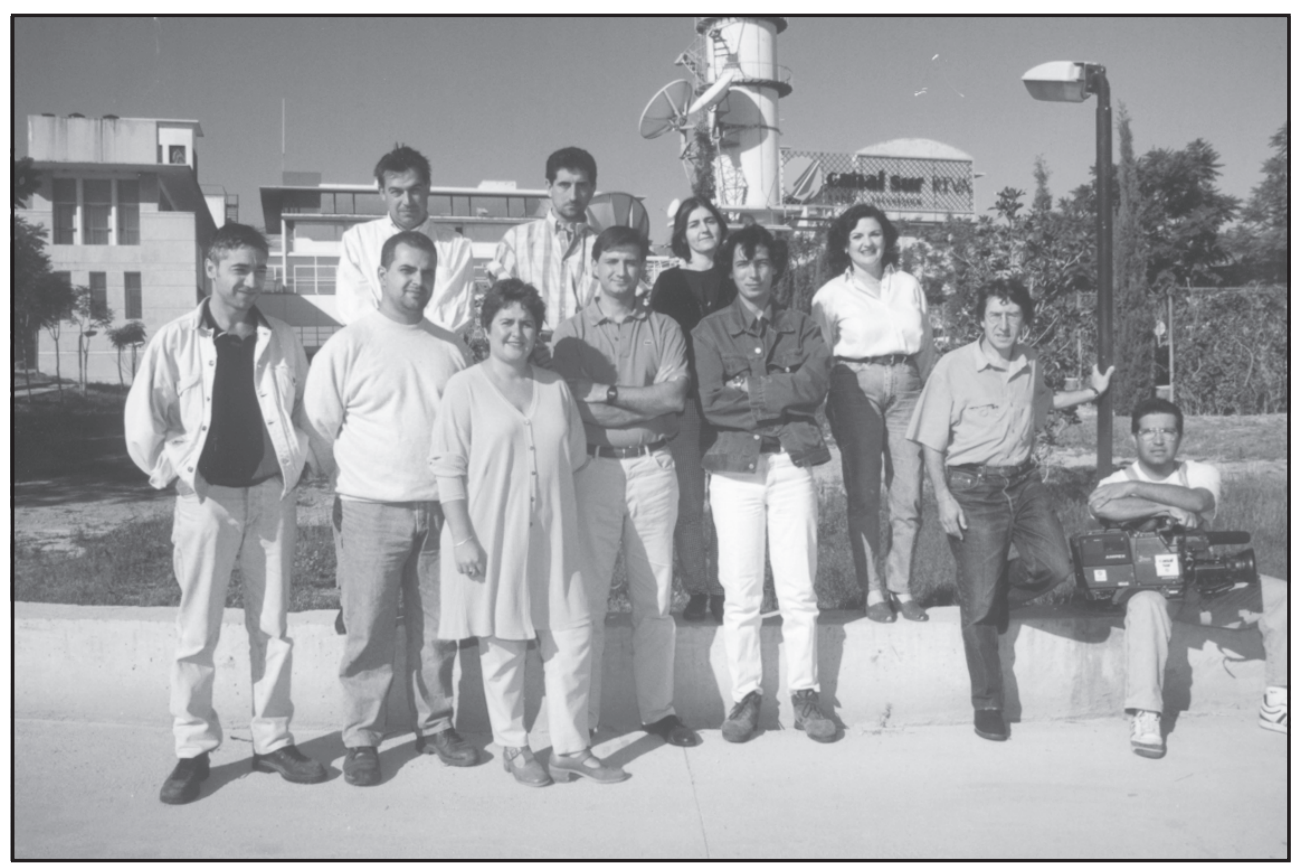

Equipo de trabajo del programa Tierra y Mar en la temporada 1997-98. De izquierda a derecha: Jorge Barrales, (arriba) Nélson López, José Manuel de Castro, Rosario González, (arriba) Jose María Domínguez, Miguel Montaño, Isabel Hernández, Francisco Olivares, Sol Varela, Ezequiel Martínez, José Gordillo.

Junto a la sección de Medio Ambiente en los Servicios Informativos diarios, esta temática es abordada de forma esporádica en los diversos programas de Canal Sur Televisión: Los Reporteros, Andalucía Directo, Parlamento o El Club de las Ideas. Este último dedica una atención especial a la educación ambiental de los niños andaluces. Sin embargo, el calificativo de "Los verdes" de Canal Sur, es atribuido por el periódico Diario 1611, al programa Tierra y Mar, espacio que cuenta con el premio Andalucía de 
Periodismo y el de La Asociación de Telespectadores de Andalucía. .c.2.3.1. En Canal Sur Televisión;

El programa Tierra y Mar surge en el mes de marzo de 1990. Durante una primera etapa, desde 1990 hasta 1992, está dedicado íntegramente a información de actualidad sobre el sector primario. A partir de esta fecha comienza una segunda etapa experimentando una evolución propiciada por tres factores: el cambio de horario de emisión, la interactividad y la incorporación de los contenidos medioambientales.

La especialización periodística en temas medioambientales en Tierra y Mar pasa por informar según tres criterios: la educación ambiental, el mantenimiento y recuperación de la biodiversidad y la presentación de modelos de conducta respetuosos con el medio ambiente, modelos que en numerosas ocasiones se hayan en la antropología social y cultural. En el gráfico número 1 se puede observar la tipología de temas sobre medio

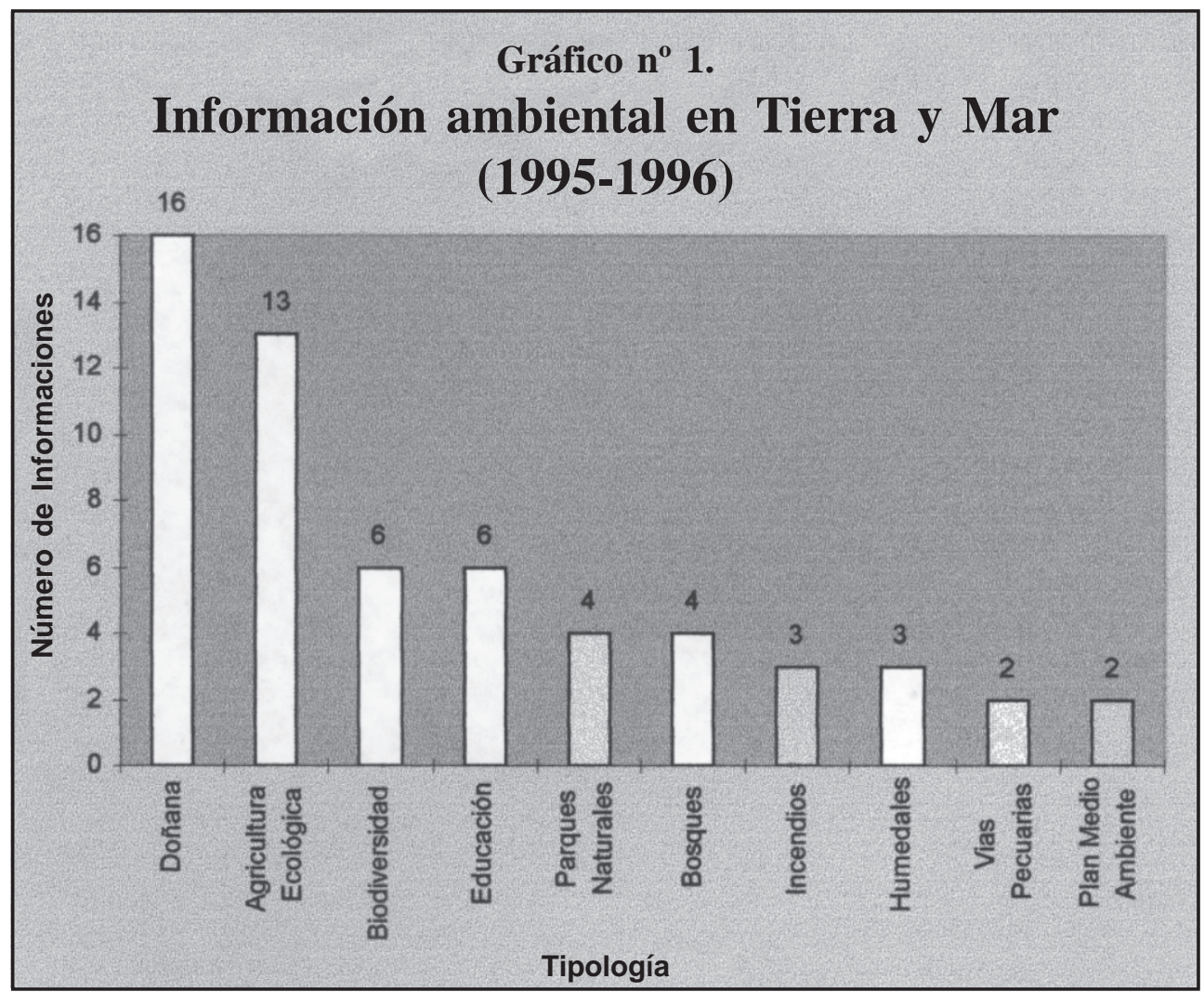

Gráfico 1. Número de informaciones y tipología en el subsector de medio ambiente durante la temporada de 1995 y 1996. Fuente: Archivo de escaletas de Tierra y Mar. Elaboración propia. 
ambiente que el programa aborda desde Septiembre de 1995 hasta el mes de Julio de 1996, fase en la que la información ambiental adquiere mayor protagonismo.

El primer lugar lo ocupa el Parque Nacional de Doñana, al que se dedican dos programas monográficos, los números 250 y 251. En segundo lugar la agricultura ecológica, a la que en esta temporada se dedica por primera vez un programa monográfico. La biodiversidad botánica y zoológica es el tercero de los ámbitos de la información ambiental. También en esta temporada se elabora, por ejemplo, el primer reportaje que se aproxima al periodismo científico y que trata sobre la enfermedad de la grafiosis en los olmos. Este reportaje fue emitido el 18 de marzo de 1996, en el programa número 252. En cuanto a especies zoológica destaca un reportaje sobre el lobo en Sierra Morena y el intento de recuperación de la biodiversidad en la Laguna de la Janda, en Cádiz. La educación ambiental también ocupa uno de los primeros puestos con la elaboración de reportajes como el dedicado al Parque Natural de los Montes de Málaga, programa número 265, emitido el 17 de Junio de 1996 o el del Jardín Botánico y Zoológico del Retiro, en Churriana, también en Málaga, emitido el 6 de Mayo de 1996 en el programa número 259. Del resto de la información ambiental destacamos la atención prestada a distintos Parques Naturales como Cazorla, programa número 256 ; Hornachuelos, programa número 252 yAlcornocales, programa número 247. En cada uno de los apartados anteriormente mencionados están presentes los rasgos de la antropología rural andaluza.

En el gráfico número 2 puede apreciarse también la evolución de la información ambiental en el programa Tierra y Mar desde Octubre de 1992 hasta Julio de 1996. Este análisis ha sido realizado mediante una base de datos en la que han sido incluidos los datos de más de trecientas escaletas -guiones- del programa. Puede observarse como durante los cuatro últimos meses del año 1992, desde Octubre a Diciembre, el programa Tierra y Mar, elabora sólo cuatro informaciones sobre esta materia. A lo largo del año 1993 la cifra se eleva hasta las dieciocho informaciones y alcanza la cifra de treinta y dos en el año 1994. La línea ascendente experimenta un leve descenso entre los meses de Enero y Junio de 1995, cuando son elaboradas veintidós informaciones de carácter medioambiental. La caída se hace más acusada entre los meses de Septiembre y Diciembre de 1995, cuando sólo se emiten nueve informaciones, la cota más baja desde 1992. Finalmente, entre Enero y Julio de 1996 la información ambiental supera esta bajada y se consolida como 


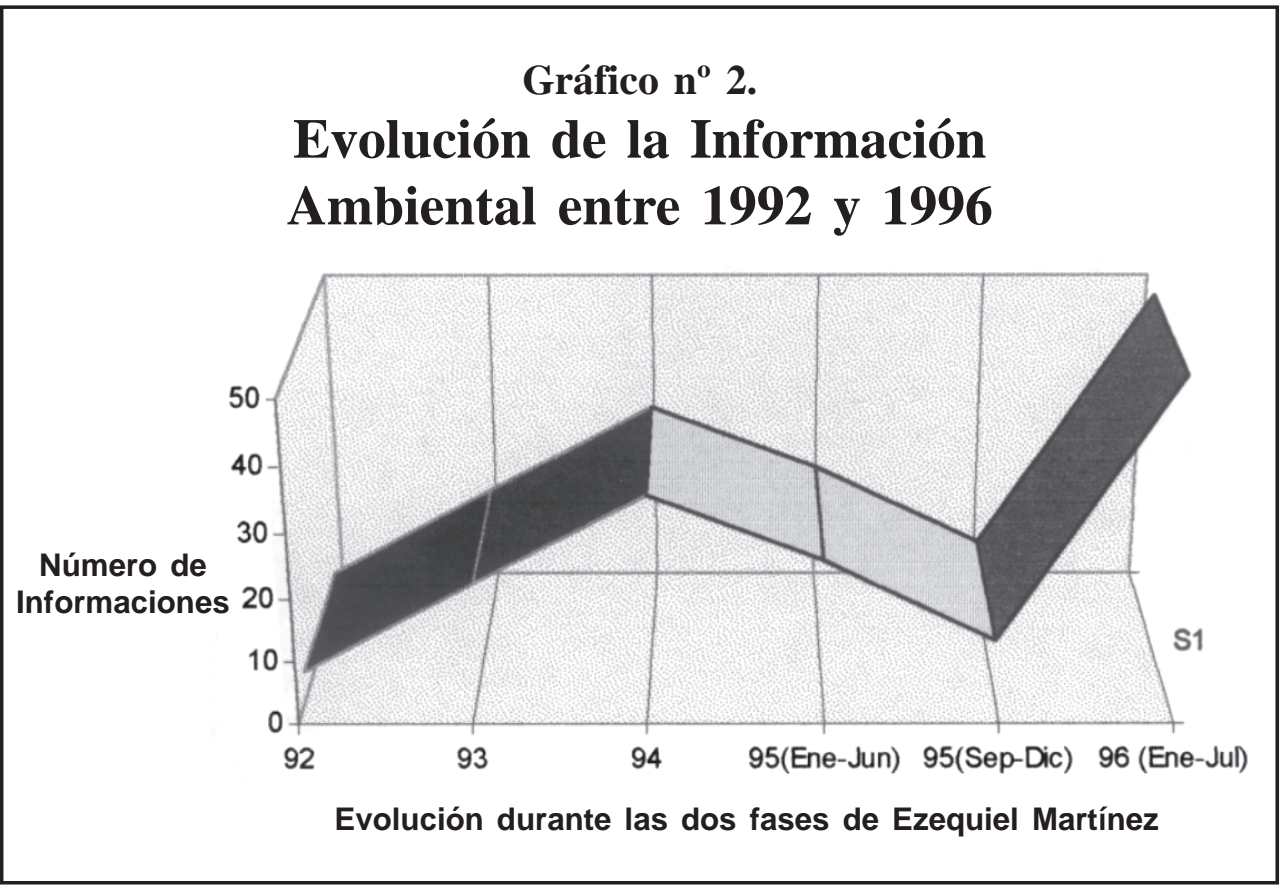

Gráfico 2. Evolución de la información ambiental en Tierra y Mar desde 1992 hasta 1996. Fuente: Archivo de escaletas de Tierra y Mar. Elaboración Propia.

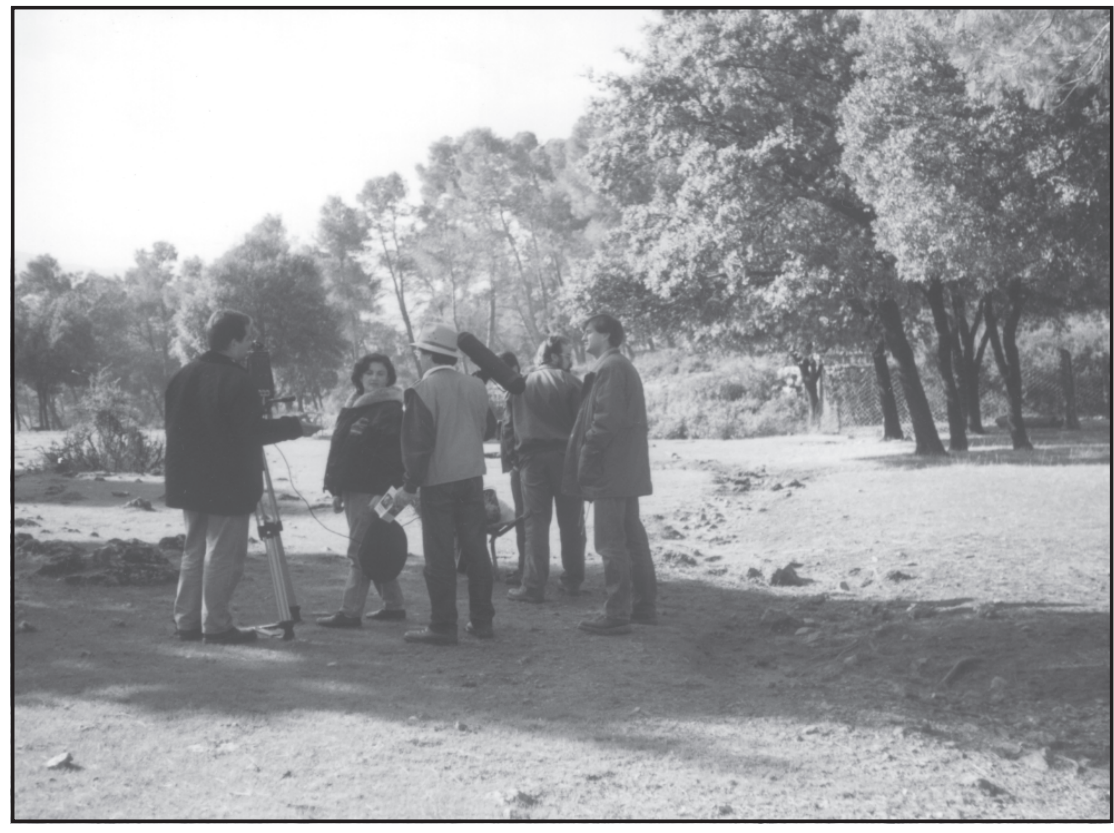

Un equipo de Tierra y Mar durante la grabación de un reportaje sobre la berrea del ciervo en el Parque Natural de Cazorla, Segura y las Villas (Jaén), en septiembre de 1996. 
la segundo subsector en materia informativa dentro del programa. En este periodo de seis meses supera todas las cifras mencionadas siendo emitidas cincuenta informaciones.

\section{Hacia una definición del concepto}

Establecido el origen histórico del periodismo ambiental, la evolución en España y Andalucía y su aparición en Canal Sur Televisión, cabe una última reflexión, la más básica. ¿Qué se entiende por periodismo ambiental?. Se puede establecer como periodismo ambiental aquél que se ocupa de la información de actualidad que contextualice, analice los procesos y enumere los efectos 12 de aquellas intervenciones relacionadas con "la naturaleza y el medio ambiente y en especial de aquellos aspectos que tienen que ver con su degradación ${ }^{13}$ ".

En la etapa contemporánea este periodismo es una opción profesional, no ideológica tal y como está siendo expresado en los más diversos foros ${ }^{14}$ : Centro de Estudios de Información Ambiental ${ }^{15}$ Instituto de Educación Continua ${ }^{16}$ y en los numerosos cursos de postgrado y master sobre información ambiental que se suceden en España en los últimos años. Esta tarea profesional se mantiene al margen del pensamiento ecológico o los movimientos sociales que luchan contra la degradación del planeta. Pero este intento de objetividad no está exenta de un cierto activismo, dado que los medios de comunicación son el único instrumento que puede educar a la escala necesaria y en el tiempo disponible ${ }^{17}$. Sólo se dispone de margen para educar a la generación actual, la que tienen en sus manos la solución a la cuestión medioambiental.

${ }^{11}$ Diario 16 de Andalucía. Lunes 10 de Febrero de 1997.

12 LEZAMIZ, Arantxa. «Presente y futuro del periodismo ambiental en España». II Congreso Nacional de Periodismo Ambiental. Madrid. Asociación de Periodistas de Información Ambiental, 1997.

${ }^{13}$ FERNÁNDEZ SÁNCHEZ, Joaquín. Periodismo Ambiental en España. Centro de Publicaciones, Secretaría General Técnica, Ministerio de Obras Públicas, Transportes y Medio Ambiente. 1995, pág. 11.

${ }^{14}$ ELCACHO, Joaquín. «El periodismo ambiental: bajo el signo de la catástrofe». II Congreso Nacional de Periodismo Ambiental. Madrid. Asociación de Periodistas de Información Ambiental, 1997.

15 VV.AA. Boletín. Centre D’estudis D’informació Ambiental. Barcelona. Noviembre 1997.

${ }^{16}$ WW.AA. «Comunicación científica. Master 1997-1998. Institut d'educación continua. 1997. Página 4.

${ }^{17}$ PÉREZ DE LAS HERAS, Mónica. «Visión internacional del periodismo ambiental». I Congreso Nacional de Periodismo Ambiental. Madrid. Asociación de Periodistas de Información Ambiental. 1996, pág. 99. 